\title{
OCR Captcha Alternative for Visually Impaired Users
}

\author{
Shivam Sehgal ${ }^{1}$ | Vandana Choudhary ${ }^{2}$ \\ ${ }^{1}$ B-tech. I.T., Maharaja Agrasen Institute of Technology, GGSIPU, New Delhi, India \\ ${ }^{2}$ Assistant Professor I.T., Maharaja Agrasen Institute of Technology, GGSIPU, New Delhi, India
}

\section{To Cite this Article}

Shivam Sehgal and Vandana Choudhary, "OCR Captcha Alternative for Visually Impaired Users", International Journal for Modern Trends in Science and Technology, 6(12): 240-247, 2020.

\section{Article Info}

Received on 10-November-2020, Revised on 02-December-2020, Accepted on 06-December-2020, Published on 10-December-2020.

\section{ABSTRACT}

The internet has been playing an increasingly important role in our daily life, with the availability of many web services such as email and search engines. However, these are often threatened by attacks from computer programs such as bots. To address this problem, CAPTCHA (Completely Automated Public Turing Test to Tell Computers and Humans Apart) was developed to distinguish between computer programs and human users. Although this mechanism offers good security and limits automatic registration to web services, but many CAPTCHAs are not user friendly and sometimes can pose some challenge to the users, especially visually impaired users. This paper provides an alternative OCR based captcha that can be used by all the users (including visually impaired users). It would provide better user experience, easy to solve and hard for a bot to crack.

KEYWORDS: CAPTCHA, Convolutional Neural Network, Handwritten Digit Recognition, Web Accessibility

\section{INTRODUCTION}

Captcha is a type of challenge-response test used in computing to determine whether or not the user is human. Traditional captchas involve finding text from the given distorted image, or finding a particular object in given images. They are super useful in determining the difference between human and bots, but they are usually poor in user experience and are difficult for visually impaired users.

The main objective of the paper is to make the captcha verification process easy for visually impaired people. Traditional CAPTCHAs provide distorted or overlapping letters and numbers that a user then has to submit via a form field. Some latest captchas involve rotating an image or finding a particular object in given set of images. These tasks seem to be trivial for a normal user but they can pose some difficulties to visually impaired users. Users having weak eyesight can find it difficult to locate the text in given image. Color blind users cannot locate the desired object in the set of images. The scope of this paper is to provide an alternative captcha system that can be used by visually impaired user as well. This captcha verification process will have a better user experience.

Idea of is to provide the user some tasks that are easy for a human to perform but are difficult/impossible for a bot. Users will be asked some basic questions like:

- What is $3+5$ ?

- Draw the smaller number: 3 or 9 .

- What is 4-3?

User will answer these questions by drawing their response on the screen. They will be provided a big canvas for drawing their response, and these questions will be read out for them using text 
reader. Their responses will be recorded and analyzed in the backend using Deep Learning algorithm. If the response is correct, then captcha In the backend, response of the user will be sent to Convolutional Neural Network (CNN) trained on handwritten characters dataset. After image preprocessing, CNN will give the results with high accuracy.

\section{- Convolutional Neural Network}

In recent years, deep learning-based techniques have been gaining significant interest in the research community for solving a variety of supervised, unsupervised and reinforcement learning problems. One of the most well-known and widely used techniques are convolutional neural networks (CNNs), a kind of neural networks which are able to automatically extract relevant features from input data. The properties

\section{METHODOLOGY}

\section{- Data Collection:}

The dataset used in MNIST dataset. The MNIST database of handwritten digits contains 60,000 training examples and 10,000 testing examples, which are $28 * 28$ images. All of digits have already been size- normalized and preprocessed and formatted (LeCun et al., 1998). The four files provided on the website are used in the training and testing for neural networks. In the process of loading the data set can be directly called from the MNIST database, but due to the

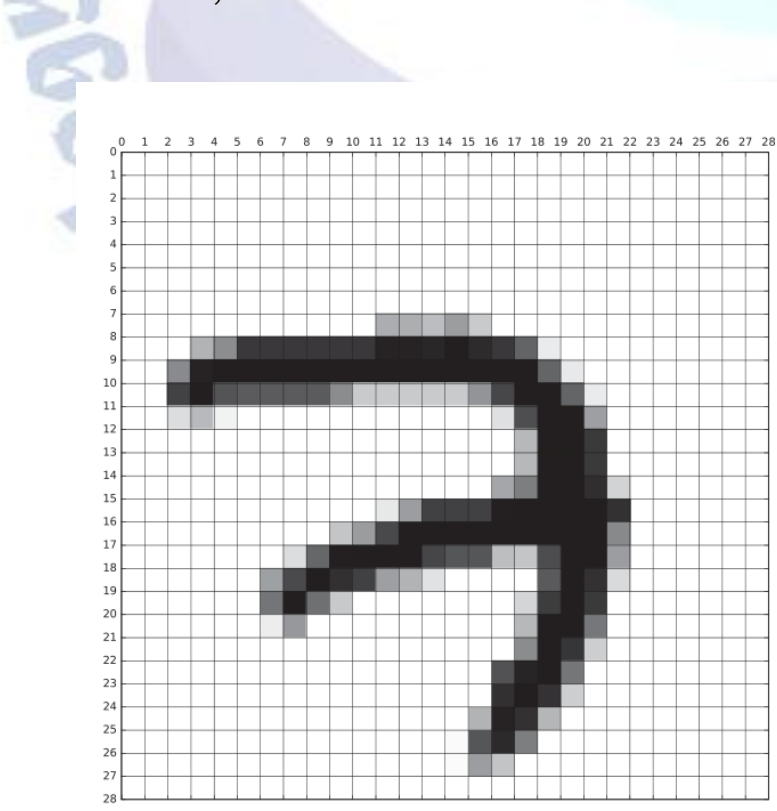

(a) MNIST sample belonging to the digit ' 7 '. verification is successful, otherwise they will be asked some other random question.

of convolutional neural networks, including the fact that they are able to retrieve features from multidimensional inputs, turn them into a very interesting alternative for solving problems within the field of computer vision. In fact, computer vision has become a testbed for validating novel techniques and innovations in CNNs.

More specifically, one dataset has been widely used for this purpose: the MNIST dataset. MNIST is a database of labeled handwritten digits, with separate training and test sets, and therefore is an easily interpretable domain that allows a fast comparison between different techniques. In fact, MNIST is so widely used that it is even used in "hello-world" tutorials of some deep learning frameworks, such as TensorFlow.

requirements of the assignment, I downloaded these image files from the website that provides the data set. Because downloading browsers may unzip these image collection files without your attention, this operation may cause the downloaded files to be larger than previously mentioned. Thus, if you need to see some problems with the original image set or data set, you can view the original site of the data set via the link provided in the reference section of the paper. Due to the use of Python's own data set, simplifying the section on data preprocessing in the code. The images are all centered in $28 * 28$ field.

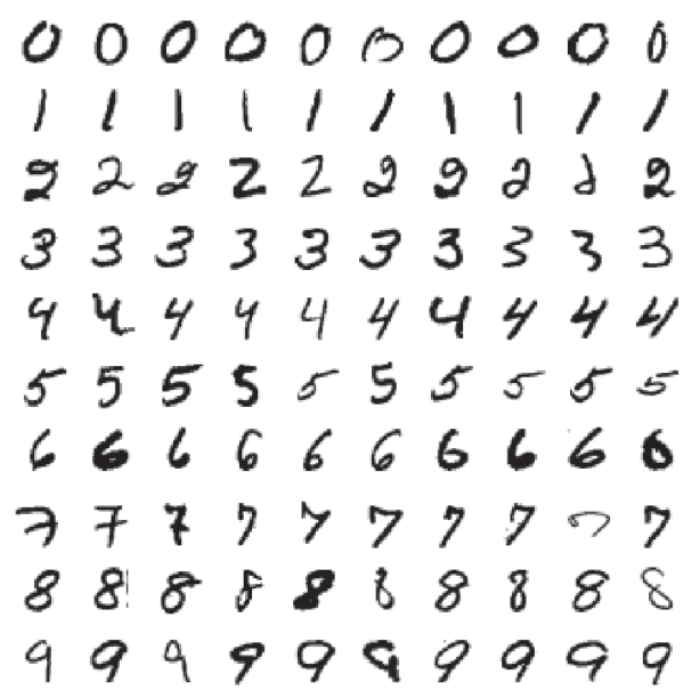

(b) 100 samples from the MNIST training set.

Figure 1. Example of the MNIST database. 


\section{○ Convolutional Neural Networks}

Due to the selection of the data set, we decompose the picture into $28^{*} 28$ blocks of the same size. According to the original trained neural network, we input a complete picture into the neural network. But for CNN, the pixel block is directly input this time. The same neural network weight will be used for every small tile. If any small tile has any abnormality, we think the tile is interested. In this neural network, there is no order in which small tiles are disturbed, and the results are still saved in the order of input. Then we will get a sequence. The part where the picture is stored is interesting. Since the array is generally large, we will first down sample it to reduce the size of the array. Find the max value in each grid square in our array. Finally, the column will be inputted into the Fully Connected Network and the neural network will determine if the picture matches.

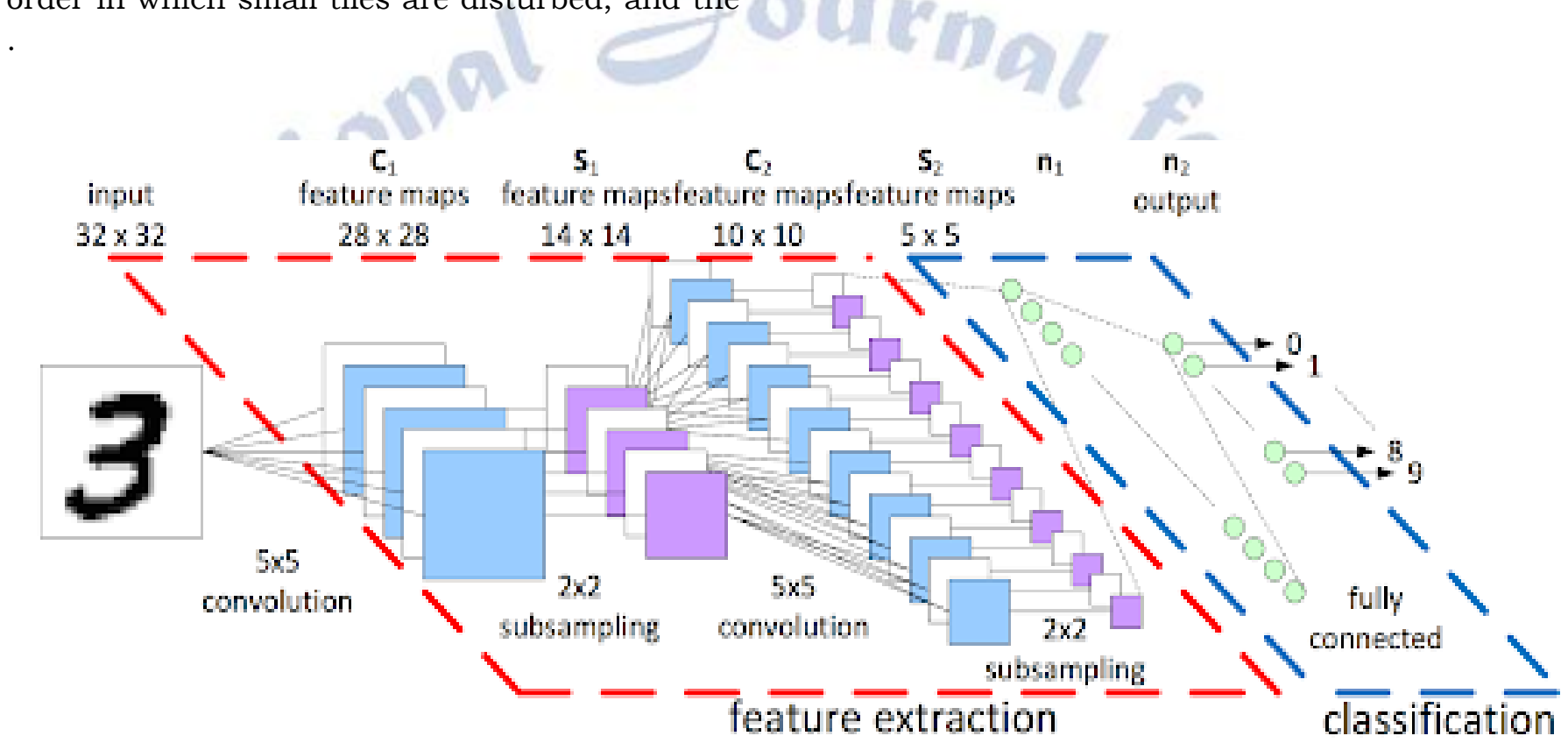

Figure 2: Convolutional Neural Network working.

\section{○ Web-application Deployment}

Flask micro-framework would be used to make to web application, for easy integration with Convolutional Neural Network in the backend. Flask Rest API would act as a bridge between frontend and the backend. User will be given some basic mathematical questions and they will draw their response on the screen (canvas). Their response will be sent to the backend, analyzed bythe deep learning model and the result will be sent back.

\section{DESIGN AND IMPLEMENTATION}

- Image Augmentation:

Image augmentation has been applied to the images to increase the size of dataset which ultimately increase the overall accuracy of the model during training.

\section{- Convolutional Neural Network using TensorFlow:}

3-layered deep Convolutional Neural Network is build using TensorFlow library. Dataset was split into three parts: Training dataset, Testing dataset and Validation dataset. CNN was trained on training dataset, and tested on validation dataset to measure the training process and prevent the overfitting on model. 

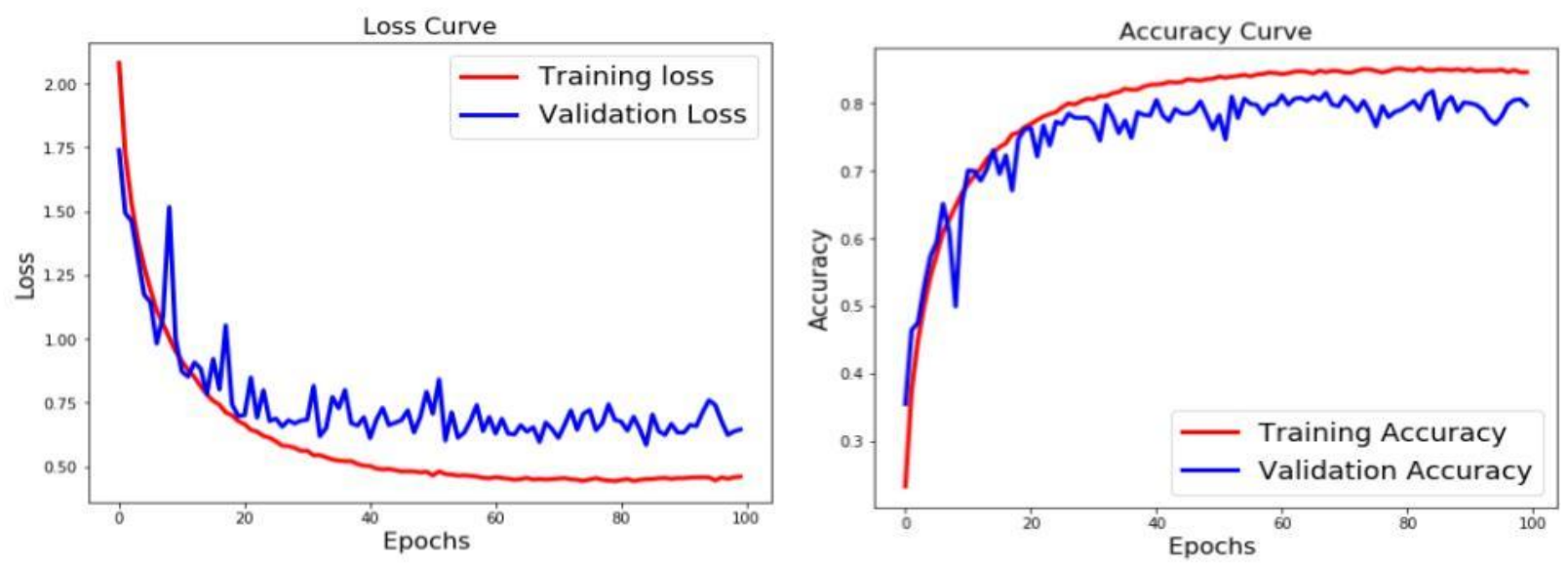

Figure 3: Convolutional Neural Network training.

Model is then tested on testing dataset to know how better it will perform on some unseen dataset.

\section{Image Preprocessing:}

The following image preprocessing would be carried out for each and every input image. First, the background of image is converted into black, and then proper cropping technique is applied to it. This will result in obtaining high accuracy on real world data.

\section{PROPOSED WORKFLOW}

Idea of is to provide the user some tasks that are easy for a human to perform but are difficult/impossible for a bot. Users will be asked some basic questions like:

- What is $3+5$ ?

- Draw the bigger number: 3 or 9?

- What is 4-3?

User will answer these questions by drawing their response on the screen. They will be provided a big canvas for drawing their response, and these questions will be read out for them using text reader. Their responses will be recorded and analyzed in the backend using the above trained Deep Learning algorithm. If the response is correct, about 20-25 minutes when trained on GPU and accuracy above $99 \%$ was achieved.

To prevent overfitting, training was stopped when validation accuracy starts decreasing.

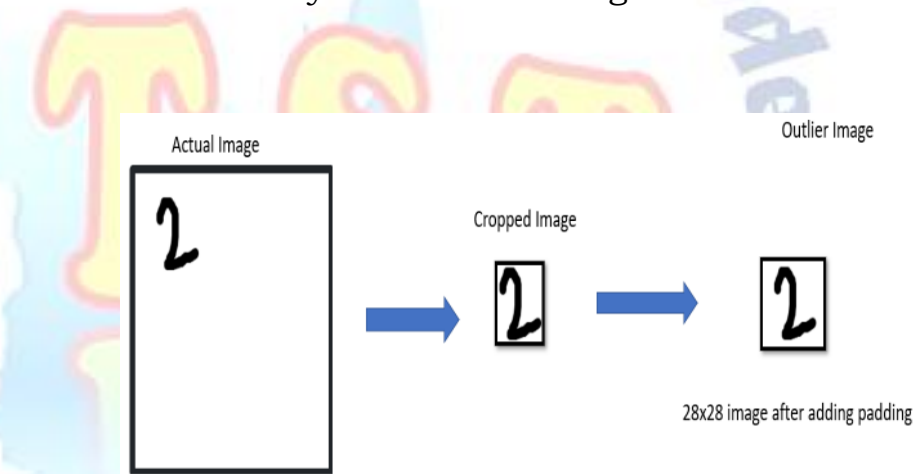

then captcha verification is successful, otherwise they will be asked some other random question.

In the backend, response of the user will be sent to Convolutional Neural Network (CNN) trained on handwritten characters dataset. After image preprocessing, CNN will give the results with high accuracy.

In order for a bot to crack this captcha verification process, first it has to understand the question given to the user. Then have to find the answer of the given question. If the correct answer is obtained by the bot, it has to draw its answer on the canvas, which is a next level challenge. Also brute-force approach will not work in order to find the answer of the given question, because the questions are generated randomly. 


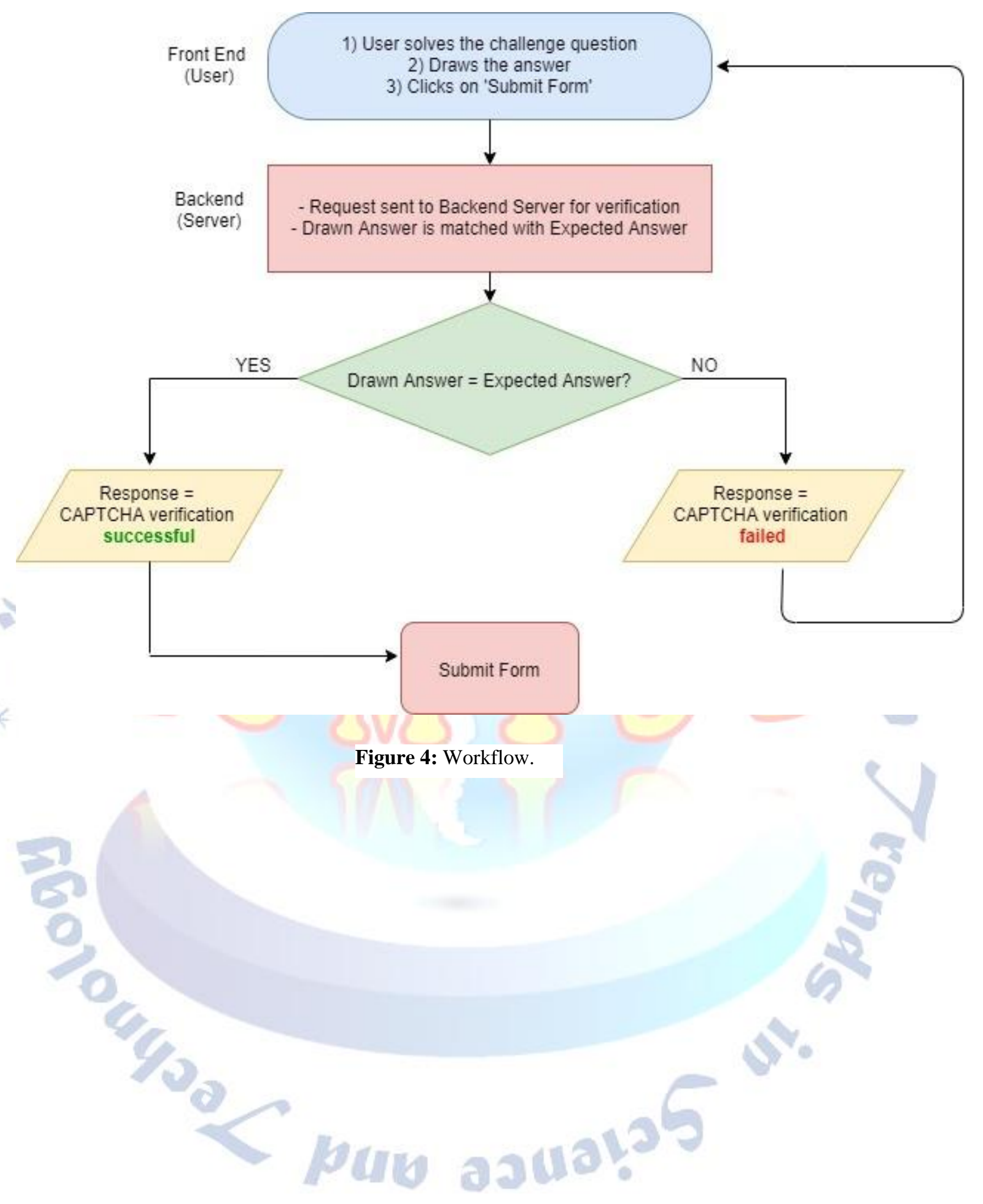




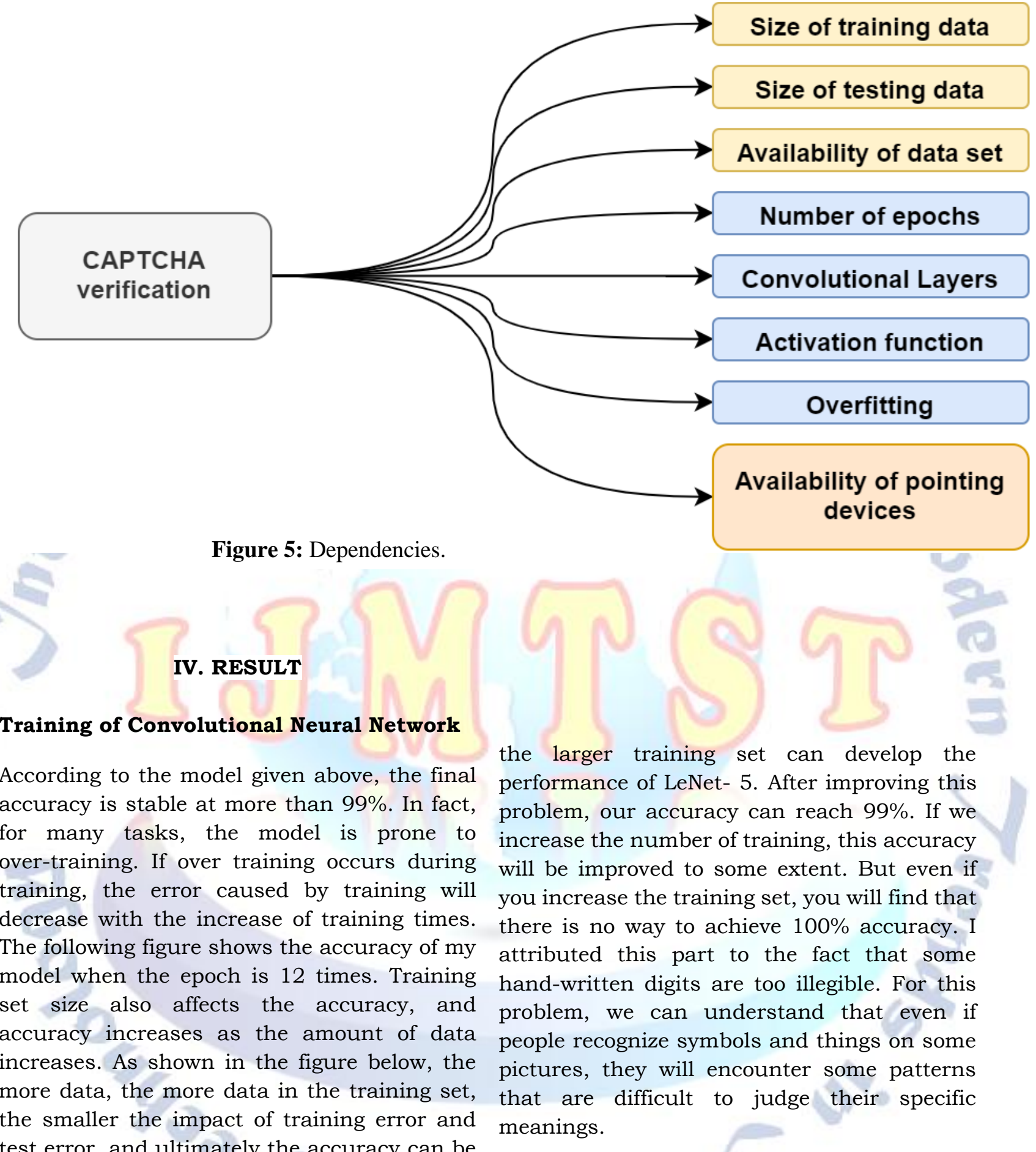
test error, and ultimately the accuracy can be improved. We can find 


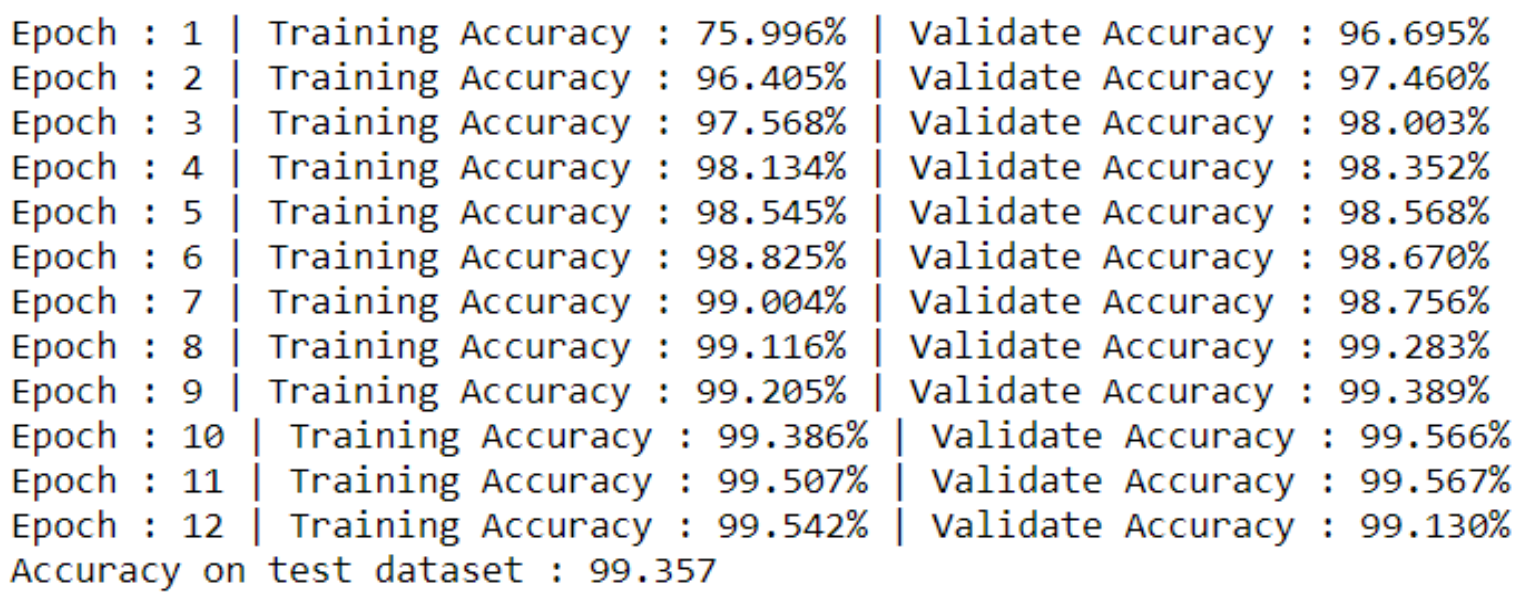

Figure 6: Training of Convolutional Neural Network.

\section{○eb Application Result}

User will be asked simple mathematical questions. These questions will be generated randomly, and user will answer them by drawing the digit on screen. User can either submit their response or try again by clearing the canvas. Also, if the captcha verification is failed, user will be asked again a new question. For a bot to crack this, it should be able to draw on canvas. It is very difficult for a bot to understand the context of the question and then draw this on screen.

When user draw their response on screen, it will be stored as an image in the backend and then analyzed by the deep learning algorithm (CNN). If the response is correct, then captcha has been verified successfully verified.
Captcha Verification :

What is the sum of 4 and 5 ?

Draw your answer inside this Box!

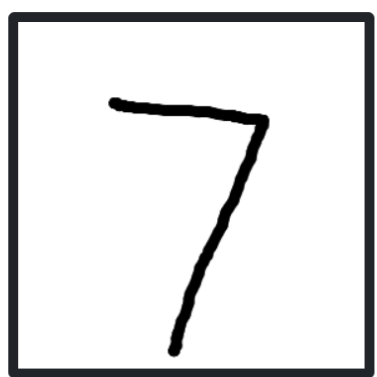

Figure 7: Web Application
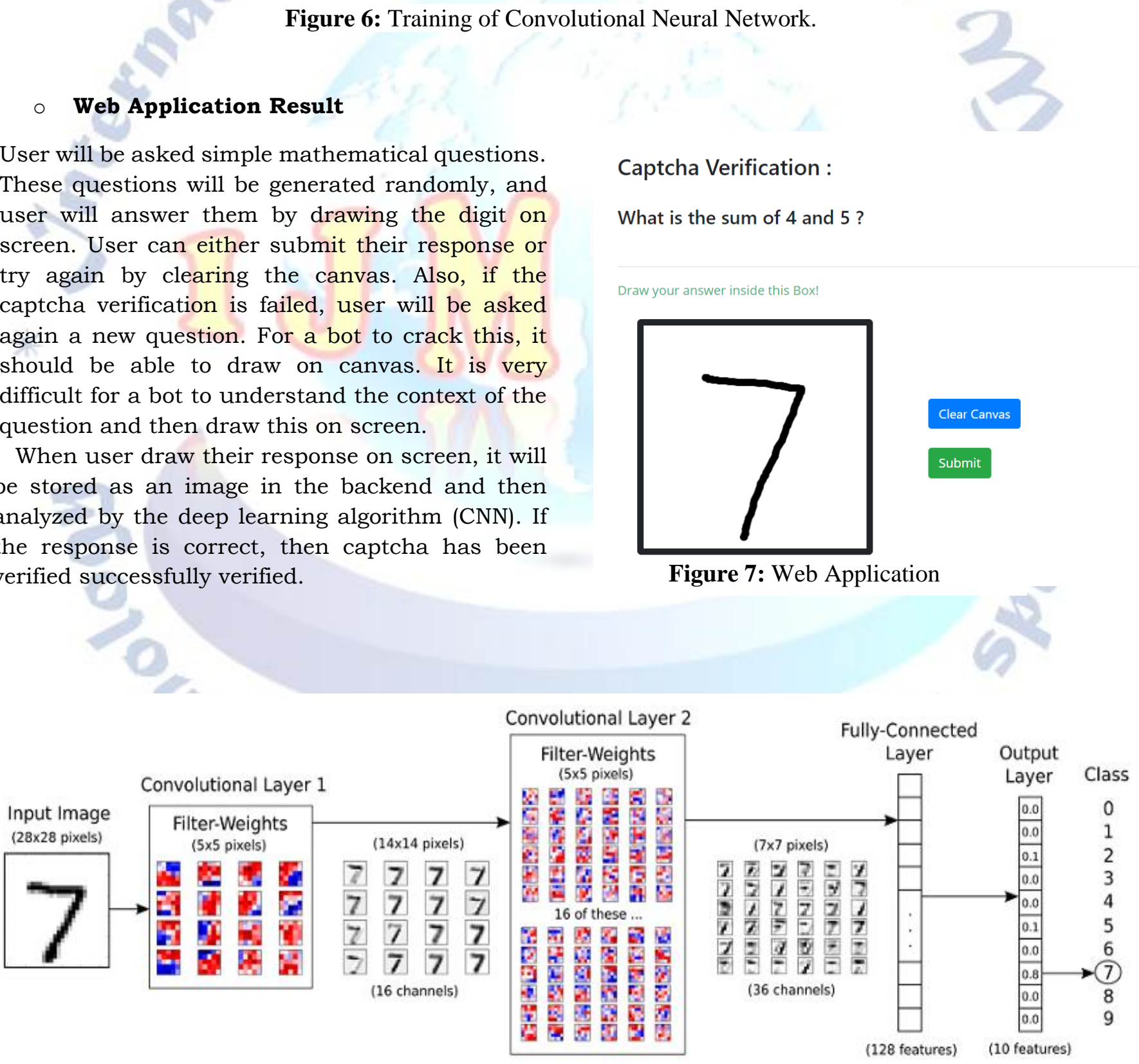

Figure 8: Working of CNN. 


\section{CONCLUSION}

The proposed captcha verification method provides overall better user experience. It can be used by visually impaired users as well. Color blind users as well as short-sighted users can find it easy to use.

This captcha is hard to crack as developing a bot that can draw something on canvas is almost impossible. Not only that, questions given to the users wouldbe completely random, hence bot has to understand the question first. Hence cracking this captcha can be extremely challenging for a bot but for the users, it would be a piece of cake.

\section{FUTURE SCOPE AND IMPROVEMENTS}

This captcha verification method is fast enough so that it can be used by all the websites for captcha verification process. Also, it provides overall better user experience.

It can be further improved by implementing following:

- RE-DRAWING ANSWER AND CHECKING SIMILARITIES IN BOTH DRAWN ANSWERS. VERY CLOSE MATCH WOULD BE REJECTED OUTRIGHT. HUMANS TEND TO HAVE VARIATIONS IN DRAWING.

- INCREASING THE DATASET BY INCLUDING ALPHABETS AS WELL.

- INCLUDING SUCH QUESTIONS THAT HAS MULTIPLE DIGIT ANSWER.

Including honeypot and other network security mechanism as secondary layers.

\section{REFERENCES}

[1] LeCun, Y., Bottou, L., Bengio, Y. \& Haffner, P. (1998). Gradient-based learning applied to document recognition. Proceedings of the IEEE, 86(11):2278-2324.

[2] LeCun, Y., Cortes, C. \& Burges, C. J. C. (1998) The MNIST database of handwritten digits [Data files].

[3] TensorFlow. MNIST for ML Beginners. 2017.

[4] LeCun, Y.; Cortes, C.; Burges, C.J.C. The MNIST Database of Handwritten Digits. 2012.

[5] Carnegie Mellon University, CAPTCHA: Telling Humans and Computers Apart.

[6] Cire,san, D.C.; Meier, U.; Masci, J.; Gambardella, L.M.; Schmidhuber, J. Flexible, High Performance Convolutional Neural Networks for Image Classification.

[7] Greg Mori, Jitendra Malik, UC Berkeley Computer Vision Group, Simon Fraser University,"Breaking a Visual CAPTCHA", accessed 15 November 2020. 